\title{
On the spectrum of noisy blown-up matrices
}

https://doi.org/10.1515/spma-2020-0010

Received October 31, 2019; accepted March 27, 2020

Abstract: We study the eigenvalues of large perturbed matrices. We consider a pattern matrix $P$, we blow it up to get a large block-matrix $B_{n}$. We can observe only a noisy version of matrix $B_{n}$. So we add a random noise $W_{n}$ to obtain the perturbed matrix $A_{n}=B_{n}+W_{n}$. Our aim is to find the structural eigenvalues of $A_{n}$. We prove asymptotic theorems on this problem and also suggest a graphical method to distinguish the structural and the non-structural eigenvalues of $A_{n}$.

Keywords: Eigenvalue, symmetric matrix, blown-up matrix, random matrix, perturbation of a matrix, singular value

MSC: 15A18, 15A52, 60F15.

\section{Introduction}

Spectral theory of random matrices has a long history (see e.g. [3], [7], [11], [12], [13] and the references therein). There are papers where the spectrum of a perturbed random matrix is studied (e.g. [11]). However, in this paper our point of view is the opposite, the random matrix is considered as a perturbation of a deterministic matrix.

Our aim is to extend the results of Bolla (see [4] and [5]). Therefore we consider a fixed deterministic pattern matrix $P$, blow it up to obtain a 'large' block-matrix $B_{n}$ and add a random noise $W_{n}$, say. We prove limit theorems for the eigenvalues of $A_{n}=B_{n}+W_{n}$ as $n \rightarrow \infty$. Our results are extensions of the results of Bolla [4] and [5] as we consider both real and complex matrices, we use several types of noise matrices, moreover we apply novel limit theorems for the random matrices. We also present numerical results and offer a graphical method to distinguish the structural and the non-structural eigenvalues.

In Section 2 and 3 we consider noise matrices with entries having zero mean values. Our results for symmetric matrices are presented in Section 2. Proposition 2.1 describes the eigenvalues of a Hermitian blown-up matrix. In Theorem 2.1 and Corollary 2.1 complex Hermitian blown-up matrices are perturbed by complex Wigner matrices. Using a known result on the eigenvalues of Wigner matrices, we prove that the structural eigenvalues of the perturbed blown-up matrix are of order $n$, while the eigenvalues 'generated' by the random noise are of order $\sqrt{n}$ almost surely. In Theorem 2.2 and Corollary 2.2 we obtain the above mentioned results for the case of perturbation with complex sample covariance matrices. In Theorem 2.3 and Corollary 2.3 real symmetric blown-up matrices are perturbed by real elliptic matrices. Applying the result of [11] on the eigenvalues of elliptic matrices, we prove that the structural eigenvalues of the perturbed blown-up matrix are of order $n$, while the other eigenvalues are of order $\sqrt{n} \log _{2} n$ almost surely.

In Section 3 non-symmetric complex matrices are considered. In Theorem 3.1 and Corollary 3.1 the asymptotic behaviour of the singular values of complex blown-up matrices perturbed by complex i.i.d. valued matrices are described.

In Section 4 we study random matrices with entries having positive mean values. Our Theorem 4.1 states that the maximal eigenvalue is 'large' when the entries of the matrix satisfy certain acceptability condition.

^Corresponding Author: István Fazekas: University of Debrecen, Faculty of Informatics, P.O. Box 400, 4002 Debrecen, Hungary, E-mail: fazekas.istvan@inf.unideb.hu

Sándor Pecsora: University of Debrecen, Faculty of Informatics, P.O. Box 400, 4002 Debrecen, Hungary 
Our result is an extension of Theorem 4.1 in [4], where symmetric random matrices having independent entries above the diagonal were considered. To prove our Theorem 4.1, we use a new Bernstein type inequality for acceptable random variables (Proposition 4.1).

In Section 5 we present numerical results. In most cases the computer experiments support our theorems. The typical behaviour of the eigenvalues of the perturbed blown-up matrix is the following. Let $\left|\lambda_{1}\right| \geq\left|\lambda_{2}\right| \geq$ $\ldots$ be the absolute values of the eigenvalues of the perturbed blown-up matrix in descending order. Then the structural eigenvalues $\left|\lambda_{1}\right| \geq\left|\lambda_{2}\right| \geq \cdots \geq\left|\lambda_{k}\right|$ are 'large' (and they rapidly decrease). The other eigenvalues $\left|\lambda_{k+1}\right| \geq\left|\lambda_{k+2}\right| \geq \ldots$ are relatively small and they decrease very slowly. So it is easy to find the structural eigenvalues. To obtain the structural eigenvalues we suggest the following numerical (graphical) procedure. Calculate some eigenvalues of $A_{n}$ starting with the largest ones in absolute value. Stop when the last 5-10 eigenvalues are close to zero and they are almost the same in absolute value. Then we obtain the increasing sequence $\left|\lambda_{t}\right| \leq\left|\lambda_{t-1}\right| \leq \cdots \leq\left|\lambda_{1}\right|$. Plot their values in the above order, then find the first abrupt change. If, say,

$$
0 \approx\left|\lambda_{t}\right| \approx\left|\lambda_{t-1}\right| \approx \cdots \approx\left|\lambda_{l+1}\right| \ll\left|\lambda_{l}\right|<\cdots<\left|\lambda_{1}\right|,
$$

that is the abrupt change is at $l$, then $\lambda_{l}, \lambda_{l-1}, \ldots, \lambda_{1}$ can be considered as the structural eigenvalues. However, there are more or less obvious exceptional cases. When the signal-noise ratio is too small or the magnitudes of the block sizes are diverse or the smallest non-zero eigenvalues of $B_{n}$ are approximately zero, then our method does not give the precise border. In these cases usually a few of the structural eigenvalues behave like the non-structural ones.

We shall denote by $\lambda_{l}(A)$ the $l$ 'th largest eigenvalue of the matrix $A . \bar{z}$ denotes the complex conjugate of the number $z$. For a random variable $\xi, \mathbb{E} \xi$ and $\mathbb{D} \xi$ stand for the expectation and the variance, respectively.

\section{Eigenvalues of perturbed symmetric matrices}

In this section we study the perturbations of Hermitian (resp. symmetric) blown-up matrices. We are interested in the eigenvalues of matrices perturbed by certain random matrices.

Let $P$ be a symmetric pattern matrix, that is a fixed complex Hermitian (in the real valued case symmetric) $k \times k$ matrix of rank $r$. Denote by $p_{i j}$ the $(i, j)$ 'th entry of $P$. Let $n_{1}, \ldots, n_{k}$ be positive integers, $n=n_{1}+\cdots+n_{k}$. Let $\tilde{B}_{n}$ be an $n \times n$ matrix consisting of $k^{2}$ blocks. Its block $(i, j)$ is of size $n_{i} \times n_{j}$ and all elements in that block are equal to $p_{i j}$. A matrix $B_{n}$ is called blown-up matrix if it can be obtained from $\tilde{B}_{n}$ by rearranging its rows and columns using the same permutation.

Following [4], we shall use the growth rate condition

$$
n \rightarrow \infty \text { so that } n_{i} / n \geq c \text { for all } i,
$$

where $c>0$ is a fixed constant. For real symmetric matrices with $0<p_{i j}<1$ for all elements, in Proposition 2.1 of [4] the following result was obtained. If (2.1) is satisfied, then $B_{n}$ has $r$ non-zero eigenvalues and $n-r$ zero eigenvalues. Moreover, all of its non-zero eigenvalues are of order $n$. Now, we extend this result to the complex valued case.

Proposition 2.1. Let $P$ be an Hermitian pattern matrix, that is a fixed complex Hermitian (in the real valued case symmetric) $k \times k$ matrix of rank $r$. Let $B_{n}$ be the blown-up matrix of $P$. Then $B_{n}$ has $r$ non-zero eigenvalues. If condition (2.1) is satisfied, then the non-zero eigenvalues of $B_{n}$ are of order $n$ in absolute value.

Proof. First we remark that the eigenvalues of $B_{n}$ are real and there is an orthonormal basis of the $n$ dimensional space consisting of eigenvectors of $B_{n}$. Let $\beta_{j}$ denote the eigenvalues and $\mathbf{u}_{j}(j=1, \ldots, n)$ the orthonormal eigenvectors of $B_{n}$. Suppose that $\beta_{1}, \ldots, \beta_{r}$ are the non-zero eigenvalues. Using the ideas of [4], we can see the following. Let $\mathbf{u}$ and $\beta$ denote a generic eigenvector-eigenvalue pair of $B_{n}$. Then for each $l=1,2, \ldots, k, n_{l}$ coordinates of $\mathbf{u}$ are equal to $u(l)$, say. Let $\tilde{\mathbf{u}}$ denote the $k$-dimensional vector having coordinates $u(1), \ldots, u(k)$ and let $N$ denote the diagonal matrix with diagonal elements $n_{1}, \ldots, n_{k}$. So the eigen- 
value equation $B_{n} \mathbf{u}=\beta \mathbf{u}$ is equivalent to $P N \tilde{\mathbf{u}}=\beta \tilde{\mathbf{u}}$. Moreover, this equation is equivalent to

$$
N^{1 / 2} P N^{1 / 2} \mathbf{v}=\beta \mathbf{v},
$$

where $\mathbf{v}=N^{1 / 2} \tilde{\mathbf{u}}$. Finally, this equation is equivalent to

$$
\tilde{N}^{1 / 2} P \tilde{N}^{1 / 2} \mathbf{v}=\frac{\beta}{n} \mathbf{v}
$$

where $\tilde{N}=\frac{1}{n} N$. We see that the vectors $\mathbf{v}_{1}, \ldots, \mathbf{v}_{r}$ are orthonormal eigenvectors of $\tilde{N}^{1 / 2} P \tilde{N}^{1 / 2}$ and the eigenvalues are $\beta_{1} / n, \ldots, \beta_{r} / n$. Now we apply the Courant-Fischer-Weyl min-max principle. First assume that the $l$ 'th eigenvalue of $P$ is positive, that is $\lambda_{l}(P)>0$. Then

$$
\lambda_{l}\left(\tilde{N}^{1 / 2} P \tilde{N}^{1 / 2}\right) \geq \min _{\mathbf{x} \in H} \frac{\mathbf{x}^{*} \tilde{N}^{1 / 2} P \tilde{N}^{1 / 2} \mathbf{x}}{\mathbf{x}^{*} \mathbf{x}} \geq \min _{j} \frac{n_{j}}{n} \min _{\mathbf{x} \in H} \frac{\mathbf{x}^{*} \tilde{N}^{1 / 2} P \tilde{N}^{1 / 2} \mathbf{x}}{\mathbf{x}^{*} \tilde{N}^{1 / 2} \tilde{N}^{1 / 2} \mathbf{x}}
$$

for any $k-l+1$ dimensional subspace $H$. As $\tilde{N}^{1 / 2}$ is a non-degenerated matrix, we obtain

$$
\lambda_{l}\left(\tilde{N}^{1 / 2} P \tilde{N}^{1 / 2}\right) \geq c \lambda_{l}(P) .
$$

On the other hand

$$
\lambda_{l}\left(\tilde{N}^{1 / 2} P \tilde{N}^{1 / 2}\right) \leq \max _{\mathbf{x} \in H} \frac{\mathbf{x}^{*} \tilde{N}^{1 / 2} P \tilde{N}^{1 / 2} \mathbf{x}}{\mathbf{x}^{*} \mathbf{x}} \leq \max _{j} \frac{n_{j}}{n} \max _{\mathbf{x} \in H} \frac{\mathbf{x}^{*} \tilde{N}^{1 / 2} P \tilde{N}^{1 / 2} \mathbf{x}}{\mathbf{x}^{*} \tilde{N}^{1 / 2} \tilde{N}^{1 / 2} \mathbf{x}}
$$

for any $l$ dimensional subspace $H$. Again, we obtain

$$
\lambda_{l}\left(\tilde{N}^{1 / 2} P \tilde{N}^{1 / 2}\right) \leq(1-c) \lambda_{l}(P) .
$$

As $\lambda_{l}\left(\tilde{N}^{1 / 2} P \tilde{N}^{1 / 2}\right)=\beta_{l} / n$, we obtain the result for positive $\lambda_{l}(P)$. If $\lambda_{l}(P)<0$, similar considerations lead to the result.

For simplicity, we assume that the rank of $P$ is $k$. We shall consider the eigenvalues in descending order, so we have $\lambda_{1}\left(B_{n}\right) \geq \cdots \geq \lambda_{n}\left(B_{n}\right)$. We know that $k$ out of the eigenvalues of $B_{n}$ are non-zero and the remaining ones are equal to zero. The $k$ non-zero eigenvalues are called structural eigenvalues of $B_{n}$. Similarly, we shall call structural eigenvalues those eigenvalues of the perturbed blown-up matrix which correspond to the structural eigenvalues of $B_{n}$. This correspondence will be described by our forthcoming theorems. We shall see that the magnitude of any structural eigenvalue is large and it is small for the other eigenvalues.

First we consider perturbations by Wigner matrices. Next theorem is a generalization of Theorem 2.3 of [4] where the real valued case and uniformly bounded perturbations were considered. In our theorem both $B_{n}$ and $W_{n}$ are complex Hermitian matrices (in particular, symmetric real matrices). The perturbation $W_{n}$ is a Wigner matrix with entries having finite 4th moment.

Theorem 2.1. Let $B_{n}, n=1,2, \ldots$, be a sequence of complex Hermitian matrices. Let the Wigner matrices $W_{n}$, $n=1,2, \ldots$, be complex Hermitian random matrices satisfying the following assumptions. Let the diagonal elements $w_{i i}$ of $W_{n}$ be i.i.d. real, let the above diagonal elements be i.i.d. complex random variables and let all of these be independent. Let $w_{i j}=\bar{w}_{j i}$ for all $i, j$. Assume that $\mathbb{E} w_{11}^{2}<\infty, \mathbb{E} w_{12}=0, \mathbb{E}\left|w_{12}-\mathbb{E} w_{12}\right|^{2}=\sigma^{2}$ is finite and positive, $\mathbb{E}\left|w_{12}\right|^{4}<\infty$. Then

$$
\limsup _{n \rightarrow \infty} \frac{\left|\lambda_{i}\left(B_{n}+W_{n}\right)-\lambda_{i}\left(B_{n}\right)\right|}{\sqrt{n}} \leq 2 \sigma
$$

for all i almost surely.

Proof. By Theorem 2.12 of [3], the largest eigenvalue of $W_{n} / \sqrt{n}$ converges to $2 \sigma$ almost surely. Now, Weyl's perturbation theorem implies (2.2). 
Corollary 2.1. Let $B_{n}, n=1,2, \ldots$, be blown-up matrices of a complex Hermitian matrix $P$ having rank $k$. Assume that condition (2.1) is satisfied. Let the Wigner matrices $W_{n}, n=1,2, \ldots$, satisfy the conditions of Theorem 2.1. Then Theorem 2.1 and Proposition 2.1 imply that $B_{n}+W_{n}$ has $k$ eigenvalues of order $n$ and the remaining eigenvalues are of order $\sqrt{n}$ almost surely.

Actually, in Theorem 2.3 of [4] the following inequality was proved (for the real valued case)

$$
\left|\lambda_{j}\left(B_{n}+W_{n}\right)-\lambda_{j}\left(B_{n}\right)\right| \leq 2 \sigma \sqrt{n}+\mathrm{O}\left(n^{1 / 3} \ln n\right)
$$

for $j=1, \ldots, n$ as $n \rightarrow \infty$ holds with probability tending to 1 (where $\sigma^{2}$ is an upper bound for the variances of the $w_{i j}$ random variables). To this end a result of [7] was applied. In the next remark we use Theorem 1.3 of [13], to improve the above remainder term.

Remark 2.1. Let $B_{n}, n=1,2, \ldots$, be a sequence of real symmetric matrices. Let the Wigner matrices $W_{n}$, $n=1,2, \ldots$, be real symmetric random matrices satisfying the following assumptions. Let $w_{i j}, 1 \leq i \leq j \leq n$, be independent (but not necessarily identically distributed). Assume that $\left|w_{i j}\right| \leq K<\infty$ for all $1 \leq i \leq j \leq n$. Let $\mathbb{E} w_{i j}=0$ and $\mathbb{E} w_{i j}^{2}=\sigma^{2}$ for all $1 \leq i<j \leq n$. Let $w_{i j}=w_{j i}$ for all $i, j$. Then

$$
\left|\lambda_{j}\left(B_{n}+W_{n}\right)-\lambda_{j}\left(B_{n}\right)\right| \leq 2 \sigma \sqrt{n}+C n^{1 / 4} \ln n
$$

for $j=1, \ldots, n$ holds with probability tending to 1 as $n \rightarrow \infty$.

Now we turn to perturbations by sample covariance matrices. The limiting behaviour of the eigenvalues of sample covariance matrices was first studied by Marchenko and Pastur [10]. Let $x_{j l}, j, l=1,2, \ldots$, be an infinite array of independent and identically distributed complex valued random variables with mean 0 and variance $\sigma^{2}$. Let $X=\left(x_{j l}\right)_{j=1,}^{n,}, \quad m=1$ be the left upper block of size $n \times m . S_{n}=\frac{1}{m} X X^{*}$ is called the sample covariance matrix.

Theorem 2.2. Let $B_{n}, n=1,2, \ldots$, be a sequence of complex Hermitian matrices. Let $S_{n}, n=1,2, \ldots$, be complex valued sample covariance matrices satisfying the above conditions. Moreover, assume that the entries of $X$ have finite fourth moments. Assume that $\lim _{n \rightarrow \infty} n / m=y \in(0, \infty)$. Then

$$
\limsup _{n \rightarrow \infty} \frac{\lambda_{i}\left(B_{n}+\sqrt{m} S_{n}\right)-\lambda_{i}\left(B_{n}\right)}{\sqrt{m}} \leq \sigma^{2}(1+\sqrt{y})^{2}
$$

for all i almost surely.

Proof. By Theorem 2.16 of [3], the largest eigenvalue of $S_{n}$ converges to $\sigma^{2}(1+\sqrt{y})^{2}$ almost surely. Now, Weyl's perturbation theorem implies the result.

Remark 2.2. As $S_{n}$ is positive semi-definite, so $0 \leq \lambda_{i}\left(B_{n}+\sqrt{m} S_{n}\right)-\lambda_{i}\left(B_{n}\right)$ is always true.

Corollary 2.2. Let $B_{n}, n=1,2, \ldots$, be blown-up matrices of a complex Hermitian matrix P having rank $k$. Assume that condition (2.1) is satisfied. Let the sample covariance matrices $S_{n}, n=1,2, \ldots$, satisfy the conditions of Theorem 2.2. Then Theorem 2.2 and Proposition 2.1 imply that $B_{n}+\sqrt{m} S_{n}$ has $k$ eigenvalues of order $n$ and the remaining eigenvalues are of order $\sqrt{n}$ almost surely.

Now we consider perturbation with matrices having independent and identically distributed elements.

Remark 2.3. Let $B_{n}, n=1,2, \ldots$, be blown-up matrices of a complex Hermitian matrix $P$ having rank $k$. Assume that condition (2.1) is satisfied. Let $v_{i j}, i, j=1,2 \ldots$, be independent identically distributed complex random variables, $i, j=1,2 \ldots$. Assume that $v_{i j}$ has zero mean, unit variance and finite fourth moment. For each $n$ let the matrix $V_{n}$ be the left upper $n \times n$ corner of the array $v_{i j}, i, j=1,2 \ldots$. Then (after labelling the eigenvalues properly)

$$
\lambda_{j}\left(B_{n}+V_{n}\right)-\lambda_{j}\left(B_{n}\right)=\mathrm{o}(1) \sqrt{n}
$$

for the non-zero eigenvalues $\lambda_{j}\left(B_{n}\right)$ of $B_{n}$, and

$$
\lambda_{j}\left(B_{n}+V_{n}\right)=\mathrm{O}(1) \sqrt{n}
$$


for the zero eigenvalues of $B_{n}$, almost surely as $n \rightarrow \infty$. This result is a simple consequence of Proposition 2.1 and the result of [12].

Now we turn to elliptic perturbations. It can be considered as a common generalization of Wigner type and i.i.d. type perturbations. We shall use condition (C0) given in [11]. The sequence of real random matrices $Y_{n}$, $n=1,2, \ldots$ satisfy condition (C0) if the following properties hold true. Let $\left(\xi_{1}, \xi_{2}\right)$ be real random variables (so called atom variables) both of them have mean zero, unit variance and finite fourth moment. Let $y_{i j}$, $i, j=1,2, \ldots$ be a double array of real random variables such that for $1 \leq i<j$ the random vectors $\left(y_{i j}, y_{j i}\right)$ are independent copies of $\left(\xi_{1}, \xi_{2}\right)$, $y_{i i}, i=1,2, \ldots$ are i.i.d. random variables with mean zero and finite variance, $\left\{y_{i i}: i=1,2, \ldots\right\} \cup\left\{\left(y_{i j}, y_{j i}\right): 1 \leq i<j\right\}$ are independent random elements. Then $Y_{n}=\left(y_{i j}\right)_{i, j=1}^{n}, n=1,2, \ldots$ are called elliptic random matrices satisfying condition (C0).

Theorem 2.3. Let $B_{n}, n=1,2, \ldots$, be a sequence of real symmetric matrices. Let $\beta_{1} \geq \beta_{2} \geq \cdots \geq \beta_{n}$ be the eigenvalues of $B_{n}$. Let $Y_{n}, n=1,2, \ldots$ be a sequence of elliptic random matrices satisfying condition (CO). Let $\left(\lambda_{1}+i \mu_{1}\right), \ldots,\left(\lambda_{n}+i \mu_{n}\right)$ be the eigenvalues of $B_{n}+Y_{n}$ so that $\lambda_{1} \geq \lambda_{2} \geq \cdots \geq \lambda_{n}($ here $i=\sqrt{-1})$. Then

$$
\limsup _{n \rightarrow \infty} \frac{\left|\lambda_{j}-\beta_{j}\right|}{\sqrt{n}\left(\log _{2} n+1.038\right)} \leq 4
$$

and

for all j almost surely.

$$
\limsup _{n \rightarrow \infty} \frac{\left|\mu_{j}\right|}{\sqrt{n}} \leq 4
$$

Proof. By Lemma 3.3 of [11] for the spectral norm we have

$$
\limsup _{n \rightarrow \infty}\left\|\frac{Y_{n}}{\sqrt{n}}\right\| \leq 4
$$

almost surely. Moreover, for non-Hermitian perturbation of a Hermitian matrix, by [9] we have the following. If $\beta_{1} \geq \beta_{2} \geq \cdots \geq \beta_{n}$ are the eigenvalues of the complex Hermitian matrix $B$ and $\left(\lambda_{1}+i \mu_{1}\right), \ldots,\left(\lambda_{n}+i \mu_{n}\right)$ are the eigenvalues of the perturbed matrix $B+Y$ so that $\lambda_{1} \geq \lambda_{2} \geq \cdots \geq \lambda_{n}$, then

$$
\left|\lambda_{j}-\beta_{j}\right| \leq\|Y\|\left(\log _{2} n+1.038\right) \text { and }\left|\mu_{j}\right| \leq\|Y\|
$$

for all $j$. So we obtain the result.

Corollary 2.3. Let $B_{n}, n=1,2, \ldots$, be blown-up matrices of a real symmetric matrix $P$ having rank $k$. Assume that condition (2.1) is satisfied. Let $Y_{n}, n=1,2, \ldots$ be a sequence of elliptic random matrices satisfying condition (CO). Then Theorem 2.3 and Proposition 2.1 imply that $B_{n}+Y_{n}$ has $k$ eigenvalues of order $n$ and the remaining eigenvalues are of order $\sqrt{n} \log _{2} n$ almost surely.

\section{Singular values of perturbed matrices}

In this section we study the perturbations of arbitrary blown-up matrices. We are interested in the singular values of matrices perturbed by certain random matrices.

Let $P$ be a pattern matrix, that is a fixed complex $a \times b$ matrix of rank $r$. Denote by $p_{i j}$ the $(i, j)$ 'th entry of $P$. Let $m_{1}, \ldots, m_{a}$ and $n_{1}, \ldots, n_{b}$ be positive integers, $m=m_{1}+\cdots+m_{a}, n=n_{1}+\cdots+n_{b}$. Let $\tilde{B}$ be an $m \times n$ matrix consisting of $m n$ blocks. Its block $(i, j)$ is of size $m_{i} \times n_{j}$ and all elements in that block are equal to $p_{i j}$. A matrix $B$ is called blown-up matrix if it can be obtained from $\tilde{B}$ by rearranging its rows and columns.

Following [5], we shall use the following growth rate condition

$$
m, n \rightarrow \infty \text { so that } m_{i} / m \geq c \text { and } n_{i} / n \geq d \text { for all } i,
$$


where $c, d>0$ are fixed constants. The following proposition is an extension of Proposition 6 of [5] to the complex valued case. The proof is similar, so we omit it.

Proposition 3.1. Let $P$ be a fixed complex $a \times b$ matrix of rank $r$. Let $B$ be the $m \times n$ blown-up matrix of $P$. If condition (3.6) is satisfied, then the non-zero singular values of $B$ are of order $\sqrt{m n}$.

Now we consider perturbations with matrices having independent and identically distributed (i.i.d.) complex entries. Let $x_{j k}, j, k=1,2, \ldots$, be an infinite array of i.i.d. complex valued random variables with mean 0 and variance $\sigma^{2}$. Let $X=\left(x_{j k}\right)_{j=1,}^{m,} \underset{k=1}{n}$ be the left upper block of size $m \times n$.

Theorem 3.1. For each $m$ and $n$ let $B=B_{m n}$ be a complex matrix of size $m \times n$ and let $X=X_{m n}$ be the above complex valued random matrix of size $m \times n$ with i.i.d. entries. Moreover, assume that the entries of $X$ have finite fourth moments. Assume that $m, n \rightarrow \infty$ so that $K_{1} \leq \frac{m}{n} \leq K_{2}$, where $0<K_{1} \leq K_{2}<\infty$ are fixed constants. Denote by $s_{i}$ and $z_{i}$ the singular values of $B$ and $B+X$, respectively, $s_{1} \geq \cdots \geq s_{\min \{m, n\}}, z_{1} \geq \cdots \geq z_{\min \{m, n\}}$. Then for all $i$

$$
\left|s_{i}-z_{i}\right|=\mathrm{O}(\sqrt{n})
$$

as $m, n \rightarrow \infty$ almost surely.

Proof. Let $S=S_{n}=\frac{1}{n} X X^{*}$ be the sample covariance matrix. By Theorem 2.16 of [3], if $\lim _{n \rightarrow \infty} m / n=y \in$ $(0, \infty)$, then the largest eigenvalue of $S_{n}$ converges to $\sigma^{2}(1+\sqrt{y})^{2}$ almost surely. Therefore the largest singular value of $\frac{1}{\sqrt{n}} X$ is bounded from above. Now, by Weyl's perturbation theorem, $\left|s_{i}-z_{i}\right| \leq\|X\|_{2}$, where $\|X\|_{2}$ is the spectral norm, that is the largest singular value of $X$. This implies the result.

Proposition 3.1 and Theorem 3.1 imply the following.

Corollary 3.1. Let $P$ be a fixed complex $a \times b$ matrix of rank $r$. Let $B=B_{m n}$ be the $m \times n$ blown-up matrix of $P$. Assume that condition (3.6) is satisfied. Let $X=X_{m n}$ be complex valued perturbation matrices satisfying the assumptions of Theorem 3.1. Denote by $z_{i}$ the singular values of $B+X, z_{1} \geq \cdots \geq z_{\min \{m, n\}}$. Then $z_{i}$ are of order $n$ for $i=1, \ldots, r$ and $z_{i}=\mathrm{O}(\sqrt{n})$ for $i=r+1, \ldots, \min \{m, n\}$ almost surely as $m, n \rightarrow \infty$ so that $K_{1} \leq \frac{m}{n} \leq K_{2}$, where $0<K_{1} \leq K_{2}<\infty$ are fixed constants.

\section{Eigenvalues of random matrices having entries with non-zero mean values}

In the previous section we considered perturbations with random matrices having zero mean entries. Now we shall show that under general conditions the eigenvalues are 'large' if the mean values are positive. Our theorem is an extension of the result of [4] where the case of independent entries were studied.

First we shall obtain a general Bernstein type inequality. For the sequence of real r.v.'s $\eta_{1}, \eta_{2}, \ldots, \eta_{n}$ we shall consider the condition

$$
\mathbb{E} e^{\sum_{i=1}^{n} t \eta_{i}} \leq \prod_{i=1}^{n} \mathbb{E} e^{t \eta_{i}}
$$

If condition (4.1) is satisfied for all $t \in \mathbb{R}$, then we are at the notion of acceptable r.v.'s, see [1]. It is known that negatively orthant dependent, negatively associated and independent random variables are acceptable. If (4.1) is true for $\eta_{1}, \eta_{2}, \ldots, \eta_{n}$, then it remains true for $\eta_{1}-a_{1}, \eta_{2}-a_{2}, \ldots, \eta_{n}-a_{n}$ for any real numbers $a_{1}, \ldots, a_{n}$, in particular for $\eta_{1}-\mathbb{E} \eta_{1}, \eta_{2}-\mathbb{E} \eta_{2}, \ldots, \eta_{n}-\mathbb{E} \eta_{n}$. Several inequalities which are known for the independent case can be easily transferred to acceptable random variables (see e.g. [6]).

Now we start with a well-known lemma. 
Lemma 4.1. Let $\eta$ be a real random variable, $|\eta| \leq C<\infty, \mathbb{E} \eta=0, \sigma^{2}=\mathbb{D} \eta$. Then

$$
\mathbb{E} e^{t \eta} \leq \exp \left\{t^{2} \sigma^{2}\left(\frac{e^{t C}-1-t C}{(t C)^{2}}\right)\right\}
$$

for any $t$.

In [2] the Berstein inequality was obtained for negatively orthant dependent sequences. Here we show that it is true in more general setting.

Proposition 4.1. For the sequence of real r.v.'s $\eta_{1}, \eta_{2}, \ldots, \eta_{n}$ assume that condition (4.1) is satisfied for all $t \geq 0$. Let $\left|\eta_{i}\right| \leq C<\infty, \mathbb{E} \eta_{i}=0, \sigma_{i}^{2}=\mathbb{D} \eta_{i}$ for all $i$. Let $\sigma^{2}=\sigma_{1}^{2}+\cdots+\sigma_{n}^{2}$. Then

$$
\mathbb{P}\left(\sum_{i=1}^{n} \eta_{i} \geq \varepsilon\right) \leq \exp \left(-\frac{\varepsilon^{2}}{2\left(\sigma^{2}+\varepsilon C / 3\right)}\right)
$$

for any $\varepsilon>0$.

Proof. We follow the usual method. By Markov's inequality, (4.1) and Lemma 4.1, we have for $t \geq 0$

$$
\mathbb{P}\left(\sum_{i=1}^{n} \eta_{i} \geq \varepsilon\right) \leq \frac{\prod_{i=1}^{n} \mathbb{E} e^{t \eta_{i}}}{e^{t \varepsilon}} \leq \exp \left\{t^{2} \sum_{i=1}^{n} \sigma_{i}^{2}\left(\frac{e^{t C}-1-t C}{(t C)^{2}}\right)-t \varepsilon\right\} .
$$

Let $t=\frac{1}{C} \log \left(1+\frac{\varepsilon C}{\sigma^{2}}\right)$. Then the above expression is equal to

$$
\exp \left(-\frac{\sigma^{2}}{C^{2}} h\left(\frac{C \varepsilon}{\sigma^{2}}\right)\right)
$$

where $h(u)=(1+u) \log (1+u)-u$. As $h(u) \geq \frac{u^{2}}{2(1+u / 3)}$ if $u \geq 0$, therefore we obtain that the above expression is less than or equal to

$$
\exp \left(-\frac{\varepsilon^{2}}{2\left(\sigma^{2}+\varepsilon C / 3\right)}\right)
$$

So we obtained the desired result.

Now, we extend Theorem 4.1 of [4] to random matrices having acceptable random variables in each row.

Theorem 4.1. Let $x_{i j}, i, j=1,2, \ldots$ be bounded non-negative real random variables, $0 \leq x_{i j} \leq K<\infty$ for each $i, j$. Assume that each row satisfies the following acceptability condition:

$$
\mathbb{E} e^{\sum_{i=1}^{n} t x_{k i}} \leq \prod_{i=1}^{n} \mathbb{E} e^{t x_{k i}}
$$

for any $t \leq 0$ and for each $k, n=1,2, \ldots$ Let $m_{k n}=\mathbb{E} x_{k 1}+\mathbb{E} x_{k 2}+\cdots+\mathbb{E} x_{k n}$ and $\sigma_{k n}^{2}=\mathbb{D} x_{k 1}+\mathbb{D} x_{k 2}+\cdots+\mathbb{D} x_{k n}$ for any $k, n$. Assume that there exist positive finite constants $c_{1}, c_{2}$, $\delta$ and $\Delta$ satisfying $0<\delta \leq \Delta \leq 1 / 2$ such that

$$
m_{k n} \geq c_{1} n^{\delta+1 / 2}, \quad \sigma_{k n}^{2} \leq c_{2} n^{\Delta+1 / 2}
$$

for all $k, n=1,2, \ldots$. Let $X_{n}=\left(x_{i j}\right)_{i, j=1}^{n}$ denote the left upper $n \times n$ part of the above infinite matrix. Then for the maximal eigenvalue we have

$$
\lambda_{\max }\left(X_{n}\right) \geq c_{1} n^{\varepsilon+1 / 2}
$$

excluding finitely many values of $n$ almost surely for any $\varepsilon$ with $0<\varepsilon<\delta$.

Proof. According to the Perron-Frobenius theorem a non-negative square matrix has an eigenvalue which is not less than the smallest row sum. Therefore

$$
\mathbb{P}\left(\lambda_{\max }\left(X_{n}\right) \geq c_{1} n^{\varepsilon+1 / 2}\right) \geq \mathbb{P}\left(\min _{i} S_{i} \geq c_{1} n^{\varepsilon+1 / 2}\right),
$$


where $S_{i}=\sum_{k=1}^{n} x_{i k}$. Now, using Proposition 4.1 and the condition $0<\varepsilon<\delta \leq \Delta \leq 1 / 2$, we obtain for the complementary event

$$
\begin{aligned}
\mathbb{P}\left(\lambda_{\max }\left(X_{n}\right)<c_{1} n^{\varepsilon+1 / 2}\right) & \leq n \mathbb{P}\left(S_{i}<c_{1} n^{\varepsilon+1 / 2}\right) \\
& \leq n \mathbb{P}\left(\mathbb{E} S_{i}-S_{i}>\mathbb{E} S_{i}-c_{1} n^{\varepsilon+1 / 2}\right) \\
& \leq n \mathbb{P}\left(\mathbb{E} S_{i}-S_{i}>c_{1} n^{1 / 2}\left(n^{\delta}-n^{\varepsilon}\right)\right. \\
& \leq n \exp \left\{-\frac{c_{1}^{2} n\left(n^{\delta}-n^{\varepsilon}\right)^{2}}{2\left(c_{2} n^{\Delta+1 / 2}+c_{1} n^{1 / 2}\left(n^{\delta}-n^{\varepsilon}\right) K / 3\right)}\right\} \\
& \leq n \exp \left\{-c_{3} n^{(-\Delta+1 / 2)}\left(n^{\delta}-n^{\varepsilon}\right)^{2}\right\} \leq n \exp \left\{-c_{4} n^{\gamma}\right\},
\end{aligned}
$$

where $\gamma>0$. So we can apply the Borel-Cantelli lemma to get that

$$
\lambda_{\max }\left(X_{n}\right)<c_{1} n^{\varepsilon+1 / 2}
$$

is satisfied only finitely often with probability 1.

\section{Numerical results}

The simulations were performed in The Julia Programming Language [8].

Example 5.1. Our first example supports Theorem 2.1 and Corollary 2.1 in the real valued case. Let $P$ be the following real symmetric pattern matrix

$$
\left[\begin{array}{llllllllll}
8 & 7 & 2 & 5 & 3 & 2 & 1 & 2 & 1 & 0 \\
7 & 9 & 6 & 3 & 4 & 0 & 2 & 1 & 2 & 1 \\
2 & 6 & 7 & 6 & 5 & 4 & 2 & 0 & 2 & 2 \\
5 & 3 & 6 & 8 & 7 & 6 & 3 & 2 & 3 & 2 \\
3 & 4 & 5 & 7 & 9 & 8 & 5 & 3 & 0 & 3 \\
2 & 0 & 4 & 6 & 8 & 7 & 6 & 4 & 3 & 1 \\
1 & 2 & 2 & 3 & 5 & 6 & 9 & 7 & 6 & 5 \\
2 & 1 & 0 & 2 & 3 & 4 & 7 & 8 & 8 & 6 \\
1 & 2 & 2 & 3 & 0 & 3 & 6 & 8 & 9 & 5 \\
0 & 1 & 2 & 2 & 3 & 1 & 5 & 6 & 5 & 7
\end{array}\right]
$$

Here the size $k=10$, the rank $r=10$, so $A_{n}$ has 10 structural eigenvalues. To obtain $B_{n}$ we blow up the matrix $P$ using block sizes $n_{1}, \ldots, n_{10}$ as follows 500, 490, 480, 470, 460, 450, 440, 430, 420, 410. Then let $W_{n}$ be a real symmetric Wigner matrix with elements $w_{i j}$ such that $w_{i j}$ are i.i.d. standard normal for $i \leq j$. We generated 1000 times $W_{n}$ and calculated the 20 largest eigenvalues of $A_{n}=B_{n}+W_{n}$ (here largest means largest in absolute value). The results are the following. 

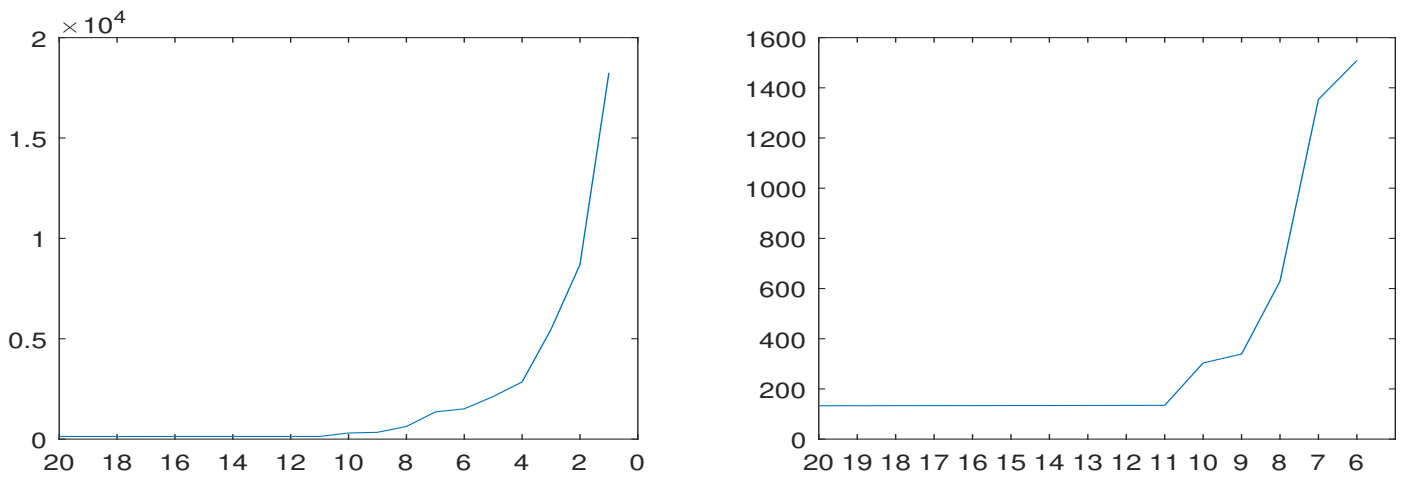

Figure 1: Left side: $\left|\lambda_{20}\left(A_{n}\right)\right|<\cdots<\left|\lambda_{1}\left(A_{n}\right)\right|$; right side: $\left|\lambda_{20}\left(A_{n}\right)\right|<\cdots<\left|\lambda_{6}\left(A_{n}\right)\right|$ in a typical realization in Example 5.1.

\begin{tabular}{rrrrr}
\hline$j$ & $\lambda_{j}\left(B_{n}\right)$ & $\lambda_{j}\left(A_{n}\right)$ & $\operatorname{mean}\left(\left|\lambda_{j}\left(A_{n}\right)\right|\right)$ & $\operatorname{var}\left(\left|\lambda_{j}\left(A_{n}\right)\right|\right)$ \\
\hline 1 & 18246.0 & 18247.0 & 18246.0 & 2.0461 \\
2 & 8692.4 & 8693.6 & 8692.9 & 2.0533 \\
3 & 5471.5 & 5472.3 & 5472.3 & 1.9234 \\
4 & 2846.3 & 2848.2 & 2847.9 & 2.0317 \\
5 & 2118.1 & 2121.0 & 2120.2 & 1.9060 \\
6 & 1504.8 & 1508.5 & 1507.8 & 2.0873 \\
7 & -1350.0 & -1353.7 & 1353.3 & 2.0347 \\
8 & -622.2 & -629.2 & 629.5 & 2.0003 \\
9 & -326.1 & -339.0 & 340.0 & 1.9204 \\
10 & 289.2 & 303.6 & 304.9 & 2.0125 \\
11 & 0 & 134.5 & 134.6 & 0.0761 \\
12 & 0 & -134.4 & 134.3 & 0.0505 \\
13 & 0 & -134.2 & 134.1 & 0.0348 \\
14 & 0 & 133.8 & 133.8 & 0.0310 \\
15 & 0 & -133.8 & 133.7 & 0.0289 \\
16 & 0 & -133.6 & 133.5 & 0.0272 \\
17 & 0 & 133.5 & 133.3 & 0.0245 \\
18 & 0 & -133.3 & 133.1 & 0.0231 \\
19 & 0 & 133.2 & 133.0 & 0.0209 \\
20 & 0 & -132.9 & 132.9 & 0.0195 \\
\hline & & & &
\end{tabular}

In the second column we listed the eigenvalues of $B_{n}$, in the third column a typical outcome (that is at a fixed experiment) for the eigenvalues of $A_{n}$, in the fourth (resp. fifth) column the averages (resp. variances) of the absolute values of the eigenvalues of $A_{n}$ using the 1000 repetitions of our experiment. We see that the variances are small, that is the experiment is stable. One can distinguish the structural and the non-structural eigenvalues because the absolute values of the non-structural eigenvalues are small and they increase very slowly, but the structural eigenvalues increase rapidly, and there is an abrupt change of the increase at the non-structural-structural border (see Figure 1, where a typical realization is presented). On the left hand side of Figure 1 we showed $\left|\lambda_{20}\left(A_{n}\right)\right|<\cdots<\left|\lambda_{1}\left(A_{n}\right)\right|$. Here one can see that the values of $\left|\lambda_{20}\left(A_{n}\right)\right|, \ldots,\left|\lambda_{11}\left(A_{n}\right)\right|$ are almost the same, and one can guess that there is a change at $\left|\lambda_{10}\left(A_{n}\right)\right|$. On the right hand side of Figure 1 we showed $\left|\lambda_{20}\left(A_{n}\right)\right|, \ldots,\left|\lambda_{6}\left(A_{n}\right)\right|$. Here (because of the new scale on the vertical axis) the abrupt change is clearly seen at $\left|\lambda_{10}\left(A_{n}\right)\right|$. So the structural eigenvalues are $\left|\lambda_{10}\left(A_{n}\right)\right|, \ldots,\left|\lambda_{1}\left(A_{n}\right)\right|$.

Example 5.2. This example also concerns Theorem 2.1 and Corollary 2.1 in the real valued case. Here the smallest non-zero eigenvalue is relatively small. So one can think that the smallest structural eigenvalue of $A_{n}$ is 

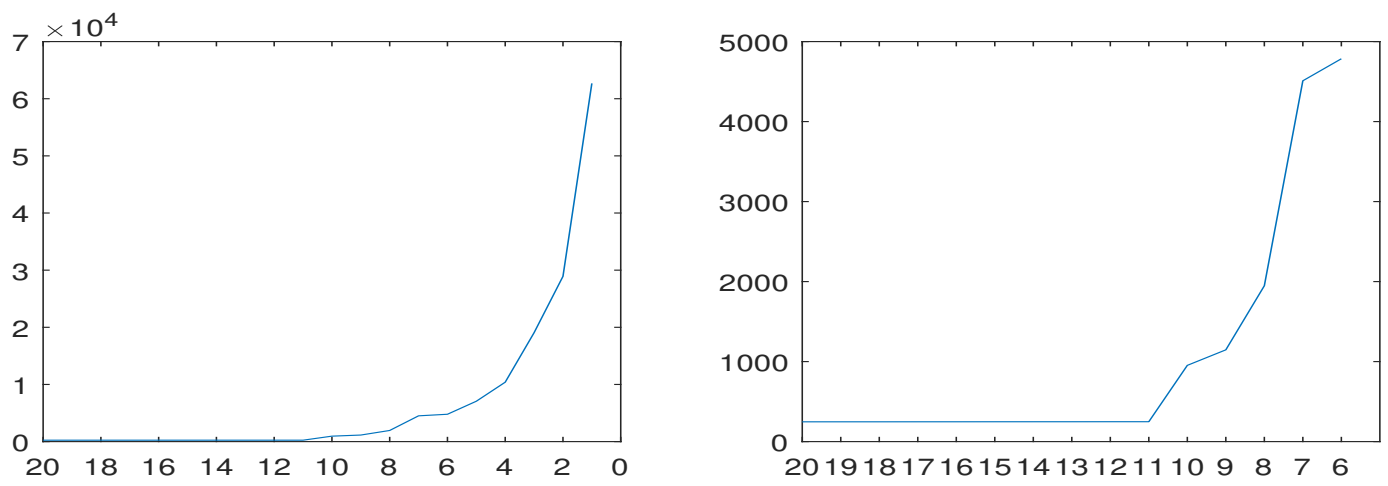

Figure 2: Left side: $\left|\lambda_{20}\left(A_{n}\right)\right|<\cdots<\left|\lambda_{1}\left(A_{n}\right)\right|$; right side: $\left|\lambda_{20}\left(A_{n}\right)\right|<\cdots<\left|\lambda_{6}\left(A_{n}\right)\right|$ in a typical realization in Example 5.2.

indistinguishable from the non-structural ones. Nevertheless, the numerical results show, that there is an abrupt change at the non-structural-structural border.

Here $P$ is a real, positive, symmetric $10 \times 10$ pattern matrix of rank 10 , the magnitudes of its elements are between 6 and 9. The eigenvalues of $P$ are

$$
\begin{array}{llllllllll}
40.22 & 19.24 & 11.72 & 6.03 & 4.73 & 3.44 & -2.91 & -1.42 & -0.70 & 0.65
\end{array}
$$

We blow up $P$ using block sizes 2000,1900, 1800, 1700, 1600, 1500, 1400,1300, 1200, 1100. The non-zero eigenvalues of the obtained blown-up matrix $B_{n}$ are the following

$$
\begin{array}{llllllllll}
62687 & 28914 & 19058 & 10393 & 7061 & -4780 & 4506 & -1938 & -1134 & 935
\end{array}
$$

We see that the last eigenvalue is relatively small for $P$ and therefore for $B_{n}$, too. The perturbation is again a real symmetric Wigner matrix $W_{n}$ with elements $w_{i j}$ such that $w_{i j}$ are i.i.d. standard normal for $i \leq j$. We calculated the first 20 eigenvalues of the perturbed matrix $A_{n}=B_{n}+W_{n}$. We performed again 1000 repetitions of the experiment. The variances of the eigenvalues were again small, so the experiment was stable. The mean values of $\left|\lambda_{6}\left(A_{n}\right)\right|, \ldots,\left|\lambda_{15}\left(A_{n}\right)\right|$ were

$\begin{array}{llllllllll}4783.8 & 4508.8 & 1945.9 & 1147.9 & 951.0 & 248.8 & 248.5 & 248.3 & 248.2 & 248.0\end{array}$

A typical realization of few largest eigenvalues of $A_{n}$ is shown on Figure 2. $\left|A_{n}(10)\right|$, i.e. the smallest structural eigenvalue of $A_{n}$ was distinguishable from the non-structural ones.

Example 5.3. This example supports Theorem 2.2 and Corollary 2.2. Let $P$ be the real symmetric pattern matrix given in Example 5.1. So there are 10 structural eigenvalues. To obtain $B_{n}$ we blow up the matrix $P$ using the same block sizes as in Example 5.1. The sample covariance matrix $S_{n}$ was generated by using real valued i.i.d. standard normal sample. We generated 1000 times $S_{n}$ and calculated the 20 largest eigenvalues of $A_{n}=$ $B_{n}+S_{n}$ (here largest means largest in absolute value). The results were stable, that is the variances were very small. The means of the 20 largest absolute values among the eigenvalues of $A_{n}$ were

$$
\begin{array}{rrrrrrrrrr}
18247.0 & 8693.3 & 5472.5 & 2847.3 & 2119.1 & 1505.8 & -1349.0 & -621.1 & -325.0 & 290.2 \\
3.9976 & 3.9785 & 3.9565 & 3.9392 & 3.9280 & 3.9253 & 3.9161 & 3.9087 & 3.8969 & 3.8905
\end{array}
$$

So we can distinguish the structural and the non-structural eigenvalues. On Figure 3 a typical realization is presented. On the left hand side of the figure we showed $\left|\lambda_{20}\left(A_{n}\right)\right|<\cdots<\left|\lambda_{1}\left(A_{n}\right)\right|$. On the right hand side of the figure we showed $\left|\lambda_{20}\left(A_{n}\right)\right|, \ldots,\left|\lambda_{6}\left(A_{n}\right)\right|$. Here the abrupt change is clearly seen at $\left|\lambda_{10}\left(A_{n}\right)\right|$. So we can decide that the structural eigenvalues are $\lambda_{1}\left(A_{n}\right), \ldots, \lambda_{10}\left(A_{n}\right)$. 

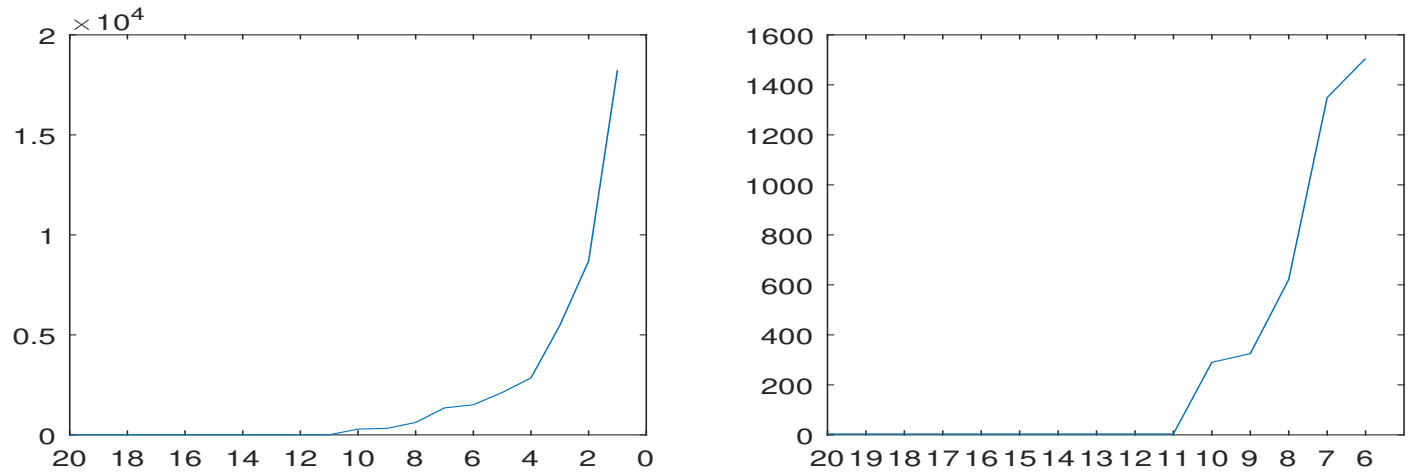

Figure 3: Left side: $\left|\lambda_{20}\left(A_{n}\right)\right|<\cdots<\left|\lambda_{1}\left(A_{n}\right)\right|$; right side: $\left|\lambda_{20}\left(A_{n}\right)\right|<\cdots<\left|\lambda_{6}\left(A_{n}\right)\right|$ in a typical realization in Example 5.3.

Example 5.4. This example supports Theorem 3.1 and Corollary 3.1. Let $P$ be the following $7 \times 8$ real nonsymmetric pattern matrix

$$
\left[\begin{array}{llllllll}
6 & 5 & 6 & 5 & 3 & 2 & 1 & 2 \\
3 & 9 & 6 & 7 & 4 & 5 & 6 & 1 \\
4 & 8 & 9 & 8 & 3 & 4 & 2 & 1 \\
5 & 7 & 6 & 8 & 7 & 5 & 3 & 2 \\
2 & 5 & 7 & 8 & 9 & 6 & 5 & 3 \\
1 & 3 & 4 & 5 & 6 & 7 & 6 & 4 \\
2 & 1 & 4 & 6 & 7 & 8 & 9 & 9
\end{array}\right]
$$

To obtain $B_{n}$ we blow up the matrix $P$ using block sizes

$$
1000,1500,1000,1200,1500,1100,1000
$$

and

$$
\text { 1000, 1000, 1200, 2000, 1100, 1000, 1100, } 1000 .
$$

The singular values of $P$ are

$$
\begin{array}{lllllll}
38.78 & 12.84 & 4.93 & 4.29 & 2.77 & 1.26 & 0.92
\end{array}
$$

The non-zero singular values of $B_{n}$ are

$\begin{array}{lllllll}48277.0 & 13495.0 & 5701.5 & 4962.5 & 3101.0 & 1407.1 & 1194.2\end{array}$

So there are 7 structural singular values in this example. We added i.i.d. standard normal noise to $B_{n}$. So we obtained matrix $A_{n}$. We calculated the singular values of $A_{n}$. Then repeated the experiment 1000 times. The mean values of the 20 largest singular values of $A_{n}$ were

$\begin{array}{rrrrrrrrrr}48277.0 & 13495.0 & 5703.0 & 4964.3 & 3103.7 & 1413.4 & 1201.6 & 187.8 & 187.5 & 187.3 \\ 187.1 & 187.0 & 186.8 & 186.7 & 186.5 & 186.4 & 186.3 & 186.1 & 186.0 & 185.9\end{array}$

We can see the abrupt change between the 7 th and 8 th singular values. The variances were small. On the left hand side of Figure 4 we showed the mean values of the largest 15 singular values of $A_{n}$. On the right hand side of Figure 4 we showed one typical outcome for the largest 15 singular values of $A_{n}$. Both figures show that the non-structural singular values are small and they are almost the same. There is an abrupt change between the non-structural and the structural singular values, and the structural singular values are large. So the numerical result supports Theorem 3.1 and Corollary 3.1.

Example 5.5. This example also concerns Theorem 3.1 and Corollary 3.1. Let here the pattern matrix be $P / 10$, where $P$ is from Example 5.4. All other parts of the experiment were the same as in Example 5.4. So there are 7 structural singular values. The non-zero singular values of $B_{n}$ were

$\begin{array}{lllllll}4827.7 & 1349.5 & 570.2 & 496.3 & 310.1 & 140.7 & 119.4\end{array}$

Here the smallest singular values seem to be far from 0 . However, the small values of the pattern matrix caused low signal-noise ratio, and so the two smallest out of the 7 structural singular values of $A_{n}$ became almost indistinguishable from the non-structural ones as the following data and figures show. 

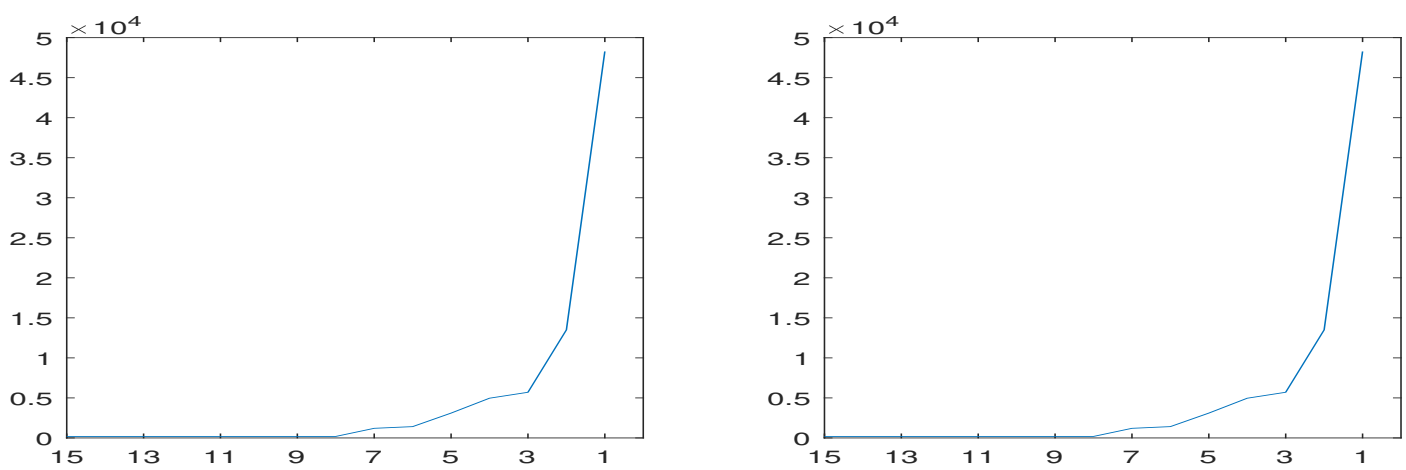

Figure 4: Left side: mean values of the largest 15 singular values of $A_{n}$; right side: one typical outcome for the largest 15 singular values of $A_{n}$ in Example 5.4.
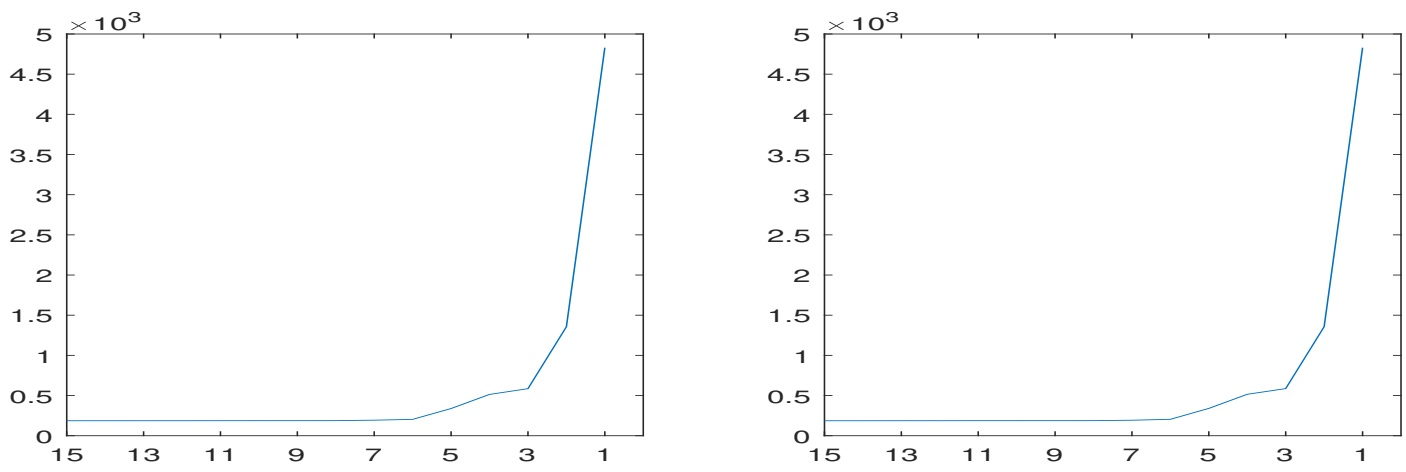

Figure 5: Left side: mean values of the largest 15 singular values of $A_{n}$; right side: one typical outcome for the largest 15 singular values of $A_{n}$ in Example 5.5.

The mean values of the 20 largest singular values of $A_{n}$ were

$\begin{array}{rrrrrrrrrr}4829.5 & 1356.0 & 585.6 & 514.0 & 338.6 & 203.6 & 193.4 & 187.8 & 187.5 & 187.3 \\ 187.1 & 186.9 & 186.8 & 186.6 & 186.5 & 186.4 & 186.2 & 186.1 & 186.0 & 185.9\end{array}$

On the left hand side of Figure 5 we showed the mean values of the largest 15 singular values of $A_{n}$ and on the right hand side one typical outcome for the largest 15 singular values of $A_{n}$. Both figures show that the non-structural singular values and the two smallest structural ones are small and they are almost the same. There is an abrupt change after the second smallest structural singular value.

Example 5.6. This example presents numerical results for Theorem 2.1 and Corollary 2.1 in the complex valued case. It shows the effect of the different block sizes on the eigenvalues of the noisy blown up matrices. We choose $P$ as a $10 \times 10$ complex Hermitian matrix with rank $r=10$, having the following eigenvalues

\section{$\begin{array}{llllllllll}9 & 8 & 7 & 6 & 5 & 4 & 3 & 2 & 1 & 0.5\end{array}$}

To create $B_{n}$, we blow up the matrix $P$ by using the method described in Section $2 . W_{n}$ is a Wigner matrix satisfying the assumptions in Theorem 2.1 and having normally distributed elements, more precisely $w_{i i}$ is real standard normal, and $w_{i j}$ is complex standard normal if $i \neq j$. Then $A_{n}=B_{n}+W_{n}$. We studied 3 different block sizes. Each case we made 1000 repetitions.

(a) In the first case we used $n_{1}=n_{2}=\cdots=n_{10}=700$. Then we calculated 20 eigenvalues of $A_{n}$ having the largest absolute values. The results are shown in the following table. Here: $\lambda_{j}\left(B_{n}\right)$ are the eigenvalues of the blown-up matrix, $\lambda_{j}\left(A_{n}\right)$ are the eigenvalues of one noisy matrix, while mean $\left(\left|\lambda_{j}\left(A_{n}\right)\right|\right)$ and $\operatorname{var}\left(\left|\lambda_{j}\left(A_{n}\right)\right|\right)$ are the means and variances of the absolute values of the 20 largest eigenvalues of $A_{n}$ : 


\begin{tabular}{rrrrr}
\hline$j$ & $\lambda_{j}\left(B_{n}\right)$ & $\lambda_{j}\left(A_{n}\right)$ & $\operatorname{mean}\left(\left|\lambda_{j}\left(A_{n}\right)\right|\right)$ & $\operatorname{var}\left(\left|\lambda_{j}\left(A_{n}\right)\right|\right)$ \\
\hline 1 & 6300 & 6300.10 & 6300.00 & 0.0019390 \\
2 & 5600 & 5599.90 & 5600.00 & 0.0033253 \\
3 & 4900 & 4900.00 & 4900.00 & 0.0040081 \\
4 & 4200 & 4200.10 & 4200.00 & 0.0025829 \\
5 & 3500 & 3500.00 & 3500.00 & 0.0036319 \\
6 & 2800 & 2800.00 & 2800.00 & 0.0026237 \\
7 & 2100 & 2100.00 & 2100.00 & 0.0024251 \\
8 & 1400 & 1400.00 & 1400.00 & 0.0029042 \\
9 & 700 & 699.98 & 700.01 & 0.0017381 \\
10 & 350 & 350.03 & 350.02 & 0.0018437 \\
11 & 0 & 2.37 & 2.37 & 0.0000100 \\
12 & 0 & 2.36 & 2.36 & 0.0000064 \\
13 & 0 & 2.35 & 2.36 & 0.0000047 \\
14 & 0 & -2.35 & 2.35 & 0.0000037 \\
15 & 0 & 2.35 & 2.35 & 0.0000028 \\
16 & 0 & 2.35 & 2.35 & 0.0000020 \\
17 & 0 & -2.34 & 2.35 & 0.0000017 \\
18 & 0 & 2.34 & 2.34 & 0.0000020 \\
19 & 0 & 2.34 & 2.34 & 0.0000015 \\
20 & 0 & -2.34 & 2.34 & 0.0000016 \\
\hline
\end{tabular}

One can see, that the 10 structural eigenvalues are well distinguishable from the non-structural ones.
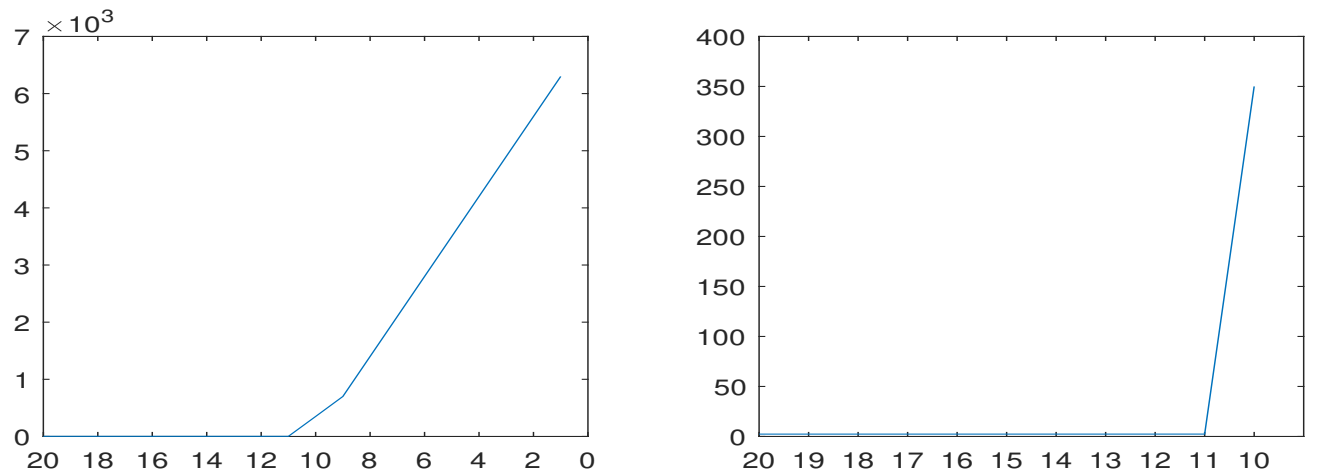

Figure 6: Left side: $\left|\lambda_{20}\left(A_{n}\right)\right|<\cdots<\left|\lambda_{1}\left(A_{n}\right)\right|$; right side: $\left|\lambda_{20}\left(A_{n}\right)\right|<\cdots<\left|\lambda_{10}\left(A_{n}\right)\right|$ in a typical realization in case (a) of Example 5.6 .

(b) In this case we used the following block sizes.

$$
n_{1}=n_{2}=560, n_{3}=n_{4}=n_{5}=n_{6}=n_{7}=n_{8}=700, n_{9}=n_{10}=840
$$

The other parts of the experiment were the same as before. This case shows the effect of different block sizes on the structural eigenvalues. We got 10 well distinguishable structural eigenvalues, but the proportion of the eigenvalues is changed. The next table shows the means of 20 eigenvalues of $A_{n}$ with the largest absolute values and Figure 7 shows the plot of this result.

$\begin{array}{rrrrrrrrrr}6705.3 & 5761.4 & 4569.6 & 4012.3 & 3694.7 & 2708.7 & 2413.9 & 1156.0 & 643.4 & 339.3 \\ 2.3666 & 2.3604 & 2.3557 & 2.3523 & 2.3500 & 2.3477 & 2.3454 & 2.3435 & 2.3415 & 2.3396\end{array}$

(c) In the last case of this example the block sizes were more diverse as in the previous cases.

$$
n_{1}=n_{2}=n_{3}=n_{4}=350, n_{5}=n_{6}=n_{7}=700, n_{8}=n_{9}=1050, n_{10}=1600
$$



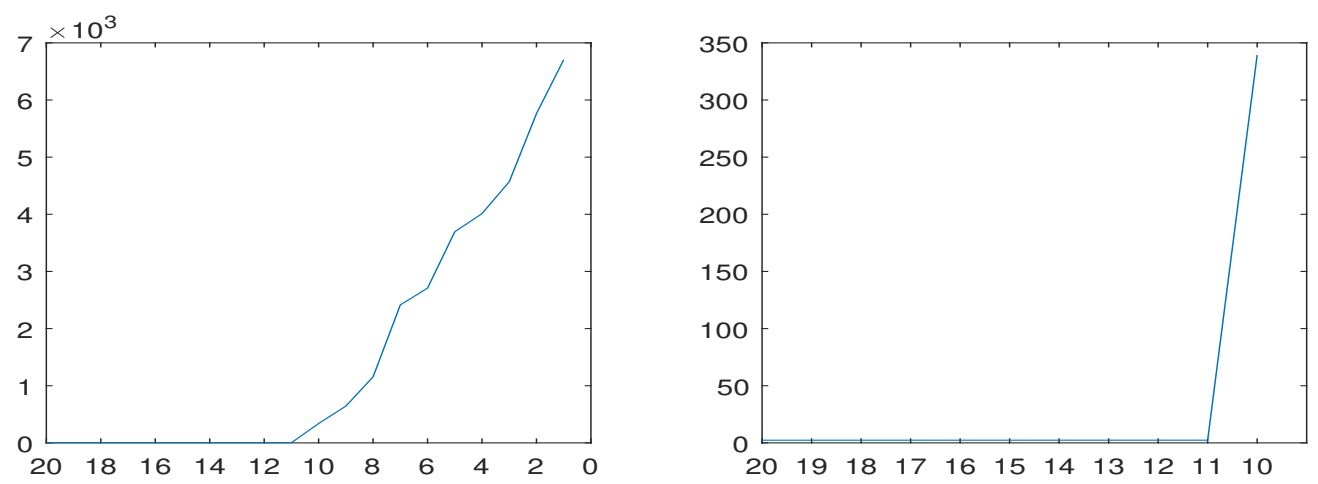

Figure 7: Left side: $\left|\lambda_{20}\left(A_{n}\right)\right|<\cdots<\left|\lambda_{1}\left(A_{n}\right)\right|$; right side: $\left|\lambda_{20}\left(A_{n}\right)\right|<\cdots<\left|\lambda_{10}\left(A_{n}\right)\right|$ in a typical realization in case (b) of Example 5.6 .

The means of the absolute values of the 20 largest eigenvalues of $A_{n}$ were

$\begin{array}{rrrrrrrrrr}10595.0 & 7150.3 & 5482.8 & 3975.3 & 2276.3 & 2075.4 & 1288.1 & 786.8 & 542.8 & 221.3\end{array}$

$\begin{array}{llllllllll}2.3671 & 2.3608 & 2.3562 & 2.3529 & 2.3505 & 2.3479 & 2.3456 & 2.3436 & 2.3414 & 2.3396\end{array}$

The above table and Figure 8 show that the structural eigenvalue with the smallest absolute value is getting closer to the non-structural ones, but it is still well distinguishable from the non-structural ones.
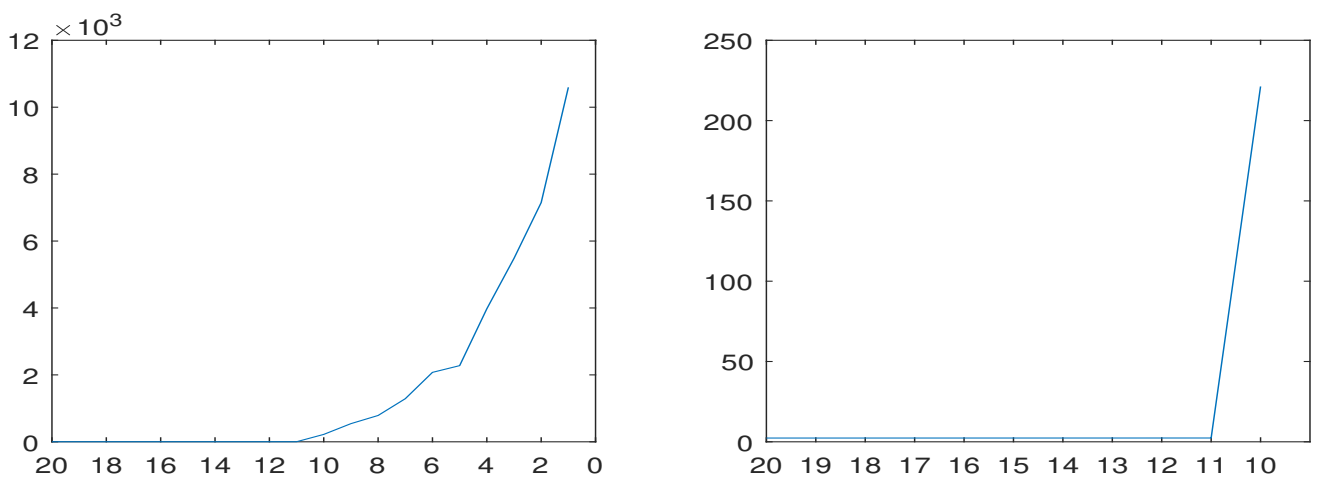

Figure 8: Left side: $\left|\lambda_{20}\left(A_{n}\right)\right|<\cdots<\left|\lambda_{1}\left(A_{n}\right)\right|$; right side: $\left|\lambda_{20}\left(A_{n}\right)\right|<\cdots<\left|\lambda_{10}\left(A_{n}\right)\right|$ in a typical realization in case (c) of Example 5.6 .

This example showed that changes in the values of $n_{1}, \ldots, n_{10}$, can cause considerable changes in the eigenvalues of the noisy blown up matrices, but the structural eigenvalues remain well distinguishable. So our method is reliable for a wide range of block sizes. 
Example 5.7. In this example we show some numerical results for Theorem 2.3 and Corollary 2.3. Let $P$ be the following matrix:

$$
P=\left[\begin{array}{llllllllll}
8 & 7 & 4 & 5 & 3 & 2 & 1 & 2 & 1 & 0 \\
7 & 9 & 6 & 3 & 4 & 3 & 2 & 1 & 2 & 1 \\
4 & 6 & 7 & 6 & 5 & 4 & 2 & 3 & 2 & 1 \\
5 & 3 & 6 & 8 & 7 & 6 & 3 & 2 & 3 & 2 \\
3 & 4 & 5 & 7 & 9 & 8 & 5 & 3 & 2 & 3 \\
2 & 3 & 4 & 6 & 8 & 7 & 7 & 5 & 3 & 1 \\
1 & 2 & 2 & 3 & 5 & 7 & 9 & 7 & 6 & 5 \\
2 & 1 & 3 & 2 & 3 & 5 & 7 & 8 & 8 & 6 \\
1 & 2 & 2 & 3 & 2 & 3 & 6 & 8 & 9 & 7 \\
0 & 1 & 1 & 2 & 3 & 1 & 5 & 6 & 7 & 8
\end{array}\right]
$$

$P$ has the following eigenvalues: $42.807,19.568,10.186,5.088,3.519,2.962,-2.543,-1.322,1.133,0.602$. This matrix is blown up with the block sizes of $n_{1}, n_{2}, \ldots, n_{10}$. The noise is real elliptic which is given as follows. Let $V_{n}$ be a matrix with independent entries which are uniformly distributed on $\left[-\frac{1}{2}\right.$; $\left.\frac{1}{2}\right]$. Let $U_{n}$ be the upper diagonal and $L_{n}$ lower diagonal part of $V_{n}$. Let $Y_{n}=V_{n}-U_{n}^{\top}+L_{n}^{\top}$ be the noise matrix (here ${ }^{\top}$ denotes transpose).

(a) In the first case the block sizes were: $n_{1}=n_{2}=\cdots=n_{10}=700$. We repeated the experiment 1000 times. After calculating the eigenvalues of the perturbed matrix, we got the following results:

\begin{tabular}{rrrrr}
\hline$j$ & $\lambda_{j}\left(B_{n}\right)$ & $\lambda_{j}\left(A_{n}\right)$ & $\operatorname{mean}\left(\left|\lambda_{j}\left(A_{n}\right)\right|\right)$ & $\operatorname{var}\left(\left|\lambda_{j}\left(A_{n}\right)\right|\right)$ \\
\hline 1 & 29965.00 & 29965.00 & 29965.00 & 0.16710 \\
2 & 13697.00 & 13698.00 & 13697.00 & 0.16797 \\
3 & 7130.20 & 7130.40 & 7130.20 & 0.19615 \\
4 & 3561.30 & 3561.40 & 3561.20 & 0.16914 \\
5 & 2463.60 & 2464.20 & 2463.60 & 0.14148 \\
6 & 2073.70 & 2073.40 & 2073.70 & 0.16437 \\
7 & -1780.10 & -1779.50 & 1780.10 & 0.15265 \\
8 & -925.42 & -924.77 & 925.45 & 0.21044 \\
9 & 793.29 & 792.95 & 793.33 & 0.18823 \\
10 & 421.12 & 420.90 & 421.10 & 0.14813 \\
11 & 0 & -34.07 & 34.28 & 0.03450 \\
12 & 0 & -34.07 & 34.18 & 0.02131 \\
13 & 0 & 33.96 & 34.09 & 0.01507 \\
14 & 0 & 33.96 & 34.04 & 0.01438 \\
15 & 0 & -33.92 & 33.98 & 0.01196 \\
16 & 0 & -33.92 & 33.94 & 0.01079 \\
17 & 0 & -33.83 & 33.90 & 0.00947 \\
18 & 0 & -33.83 & 33.86 & 0.00875 \\
19 & 0 & 33.67 & 33.83 & 0.00838 \\
20 & 0 & -33.64 & 33.80 & 0.00816 \\
\hline & & & &
\end{tabular}

Figure 9 shows the result of one fixed experiment:

(b) In this case the pattern matrix $P$ and the method to generate $Y_{n}$ were not changed, but we used diverse block sizes, $n_{1}=n_{2}=560, n_{3}=\cdots=n_{8}=700, n_{9}=n_{10}=840$. Now the eigenvalues of $A_{n}$ in a typical realization were the following:

$\begin{array}{rrrrrrrrrr}30422.0 & 13767.0 & 6666.7 & 3594.9 & 2461.2 & 1906.1 & 1738.8 & 931.1 & 818.9 & 432.5 \\ 34.27 & 34.18 & 34.07 & 34.03 & 33.97 & 33.93 & 33.89 & 33.86 & 33.83 & 33.80\end{array}$

Figure 10 shows the graphical visualization of the eigenvalues. 

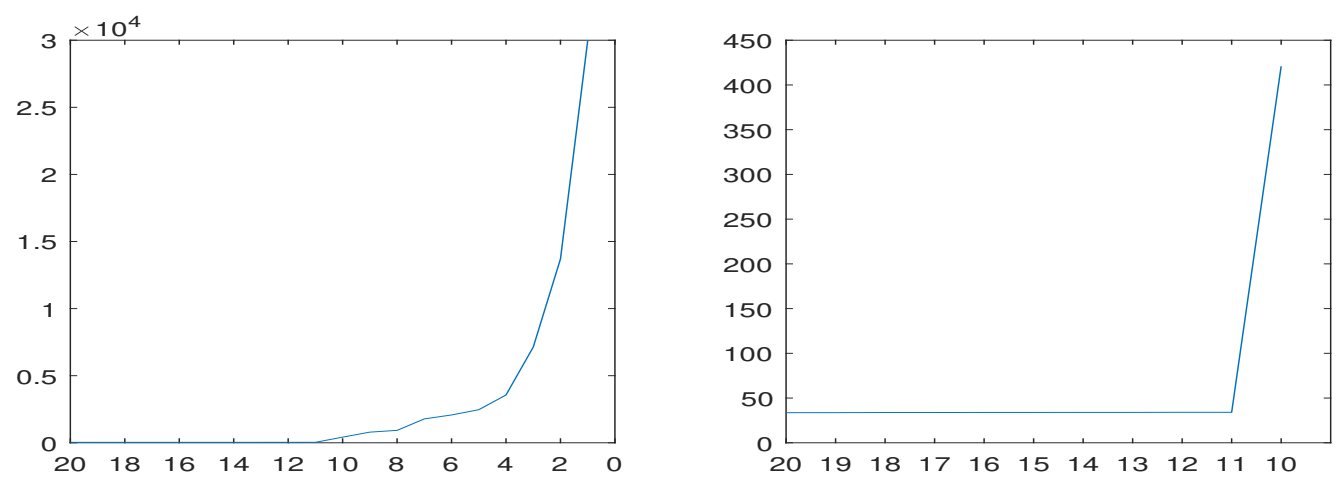

Figure 9: Left side: $\left|\lambda_{20}\left(A_{n}\right)\right|<\cdots<\left|\lambda_{1}\left(A_{n}\right)\right|$; right side: $\left|\lambda_{20}\left(A_{n}\right)\right|<\cdots<\left|\lambda_{10}\left(A_{n}\right)\right|$ in a typical realization in case (a) of Example 5.7 .
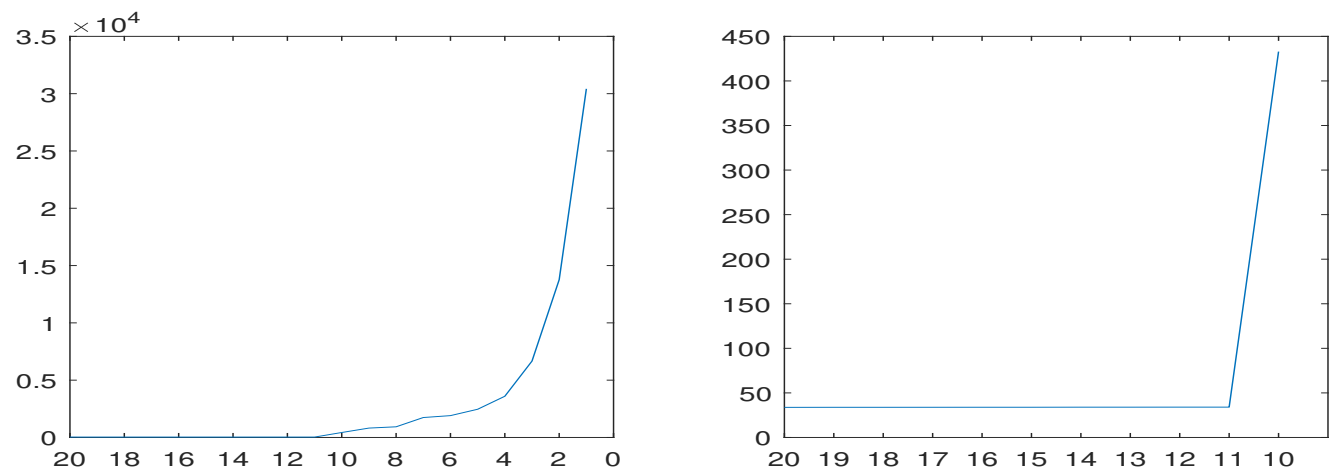

Figure 10: Left side: $\left|\lambda_{20}\left(A_{n}\right)\right|<\cdots<\left|\lambda_{1}\left(A_{n}\right)\right|$; right side: $\left|\lambda_{20}\left(A_{n}\right)\right|<\cdots<\left|\lambda_{10}\left(A_{n}\right)\right|$ in a typical realization in case (b) of Example 5.7.

(c) In this case the pattern matrix $P$ and the method we used to generate $Y_{n}$ were not changed compared to the previous cases, the only change is in the sizes of the blocks. Now, the block sizes are the following: $n_{1}=\cdots=n_{9}=770, n_{10}=70$ witch means, that in the blown-up matrix the first nine rows of blocks contain nine blocks of size $770 \times 770$ and one block of size $770 \times 70$, the last row contains nine blocks of size $70 \times 770$ and one block of size $70 \times 70$. The result of this case is presented in the following table and Figure 11. 


\begin{tabular}{rrrrr}
\hline$j$ & $\lambda_{j}\left(B_{n}\right)$ & $\lambda_{j}\left(A_{n}\right)$ & $\operatorname{mean}\left(\left|\lambda_{j}\left(A_{n}\right)\right|\right)$ & $\operatorname{var}\left(\left|\lambda_{j}\left(A_{n}\right)\right|\right)$ \\
\hline 1 & 31351.00 & 31351.00 & 31351.00 & 0.14696 \\
2 & 13162.00 & 13161.00 & 13162.00 & 0.15903 \\
3 & 7443.40 & 7443.00 & 7443.40 & 0.17163 \\
4 & 3503.30 & 3502.30 & 3503.30 & 0.16403 \\
5 & 2369.30 & 2368.90 & 2369.30 & 0.18241 \\
6 & -1773.90 & -1773.80 & 1773.80 & 0.14494 \\
7 & 1311.70 & 1311.90 & 1311.80 & 0.16097 \\
8 & -726.44 & -727.34 & 726.47 & 0.18760 \\
9 & 655.79 & 655.85 & 655.83 & 0.17405 \\
10 & 244.50 & 245.32 & 244.47 & 0.15170 \\
11 & 0 & -34.15 & 34.27 & 0.02765 \\
12 & 0 & -34.10 & 34.17 & 0.01817 \\
13 & 0 & -34.10 & 34.10 & 0.01521 \\
14 & 0 & 34.08 & 34.03 & 0.01171 \\
15 & 0 & 34.08 & 33.98 & 0.00865 \\
16 & 0 & -33.94 & 33.94 & 0.00766 \\
17 & 0 & -33.94 & 33.90 & 0.00737 \\
18 & 0 & 33.91 & 33.86 & 0.00711 \\
19 & 0 & 33.91 & 33.83 & 0.00747 \\
20 & 0 & 33.83 & 33.79 & 0.00727 \\
\hline
\end{tabular}
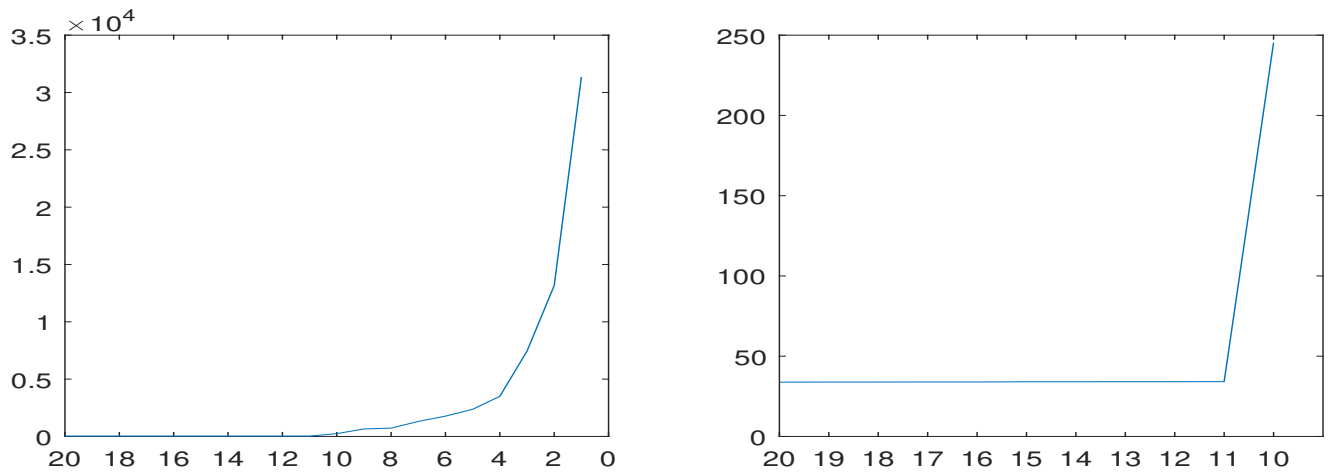

Figure 11: Left side: $\left|\lambda_{20}\left(A_{n}\right)\right|<\cdots<\left|\lambda_{1}\left(A_{n}\right)\right|$; right side: $\left|\lambda_{20}\left(A_{n}\right)\right|<\cdots<\left|\lambda_{10}\left(A_{n}\right)\right|$ in a typical realization in case (c) of Example 5.7.

This example showed that changes in the values of $n_{1}, \ldots, n_{10}$, can cause changes in the eigenvalues of the noisy blown up matrices, but the structural eigenvalues remain well distinguishable. So our method is reliable for a wide range of block sizes.

\section{Conclusion}

In the case when the perturbation matrix has zero mean entries, then our theoretical results show that the structural eigenvalues are 'large' and the non-structural ones are 'small'. Our numerical results give insight into behaviour of the sequence of eigenvalues. Let $\left|\lambda_{1}\right| \geq\left|\lambda_{2}\right| \geq \ldots$ be the absolute values of the eigenvalues of the perturbed blown-up matrix in descending order. Then the structural eigenvalues $\left|\lambda_{1}\right| \geq\left|\lambda_{2}\right| \geq \cdots \geq\left|\lambda_{l}\right|$ 
are 'large' and they rapidly decrease. The other eigenvalues $\left|\lambda_{l+1}\right| \geq\left|\lambda_{l+2}\right| \geq \ldots$ are relatively small and they decrease very slowly. So it is easy to find the structural eigenvalues. To obtain the structural eigenvalues we suggest the following numerical (graphical) procedure. Calculate some eigenvalues of $A_{n}$ starting with the largest ones in absolute value. Stop when the last 5-10 eigenvalues are close to zero and they are almost the same in absolute value. Then we obtain the increasing sequence $\left|\lambda_{t}\right| \leq\left|\lambda_{t-1}\right| \leq \cdots \leq\left|\lambda_{1}\right|$. Plot their values in the above order, then find the first abrupt change. If, say,

$$
0 \approx\left|\lambda_{t}\right| \approx\left|\lambda_{t-1}\right| \approx \cdots \approx\left|\lambda_{l+1}\right| \ll\left|\lambda_{l}\right|<\cdots<\left|\lambda_{1}\right|,
$$

that is the abrupt change is at $l$, then $\lambda_{l}, \lambda_{l-1}, \ldots, \lambda_{1}$ can be considered as the structural eigenvalues. The typical abrupt change after the non-structural eigenvalues can be seen on Figure 12. Similar method is valid for the singular values, too.

Our results are stable in the sense that variances are small and the behaviour of the eigenvalues is the same in wide range of conditions. Our method does not work only in extreme circumstances (the signal-noise ratio is too small, the smallest eigenvalue of the pattern matrix is approximately zero, or the block sizes are very diverse).

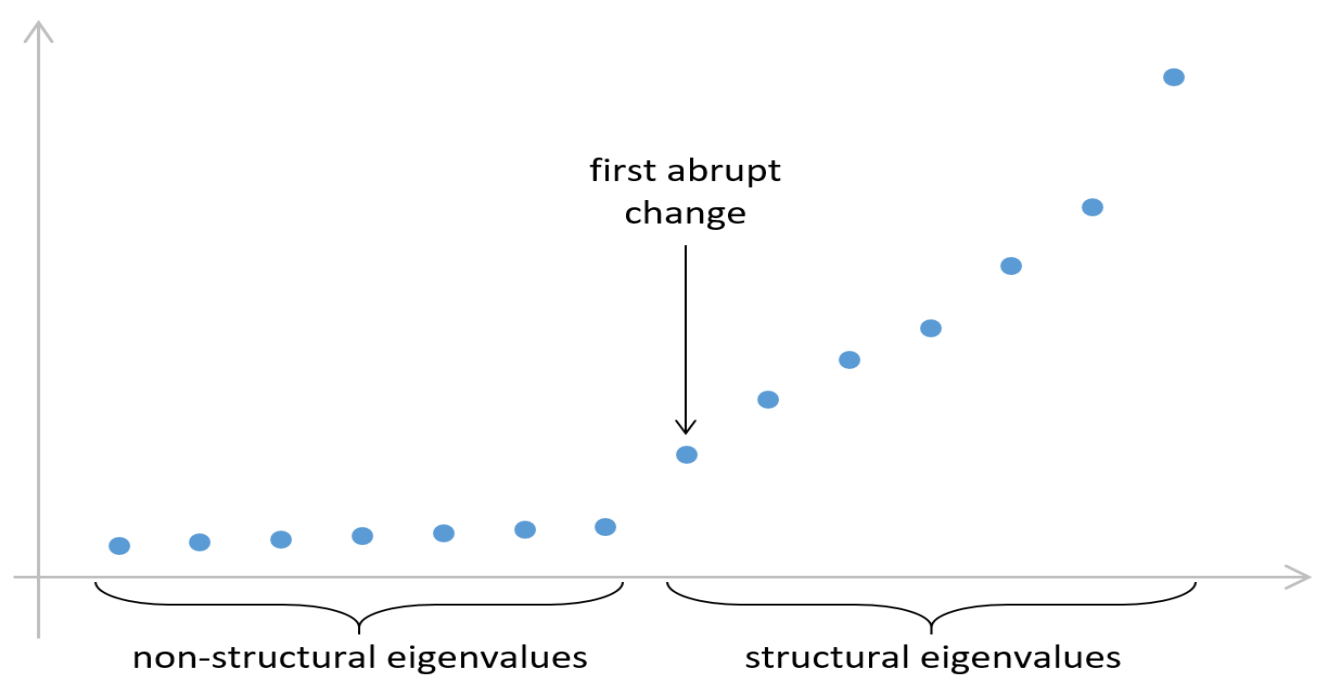

Figure 12: The abrupt change after the non-structural eigenvalues

Acknowledgement: This work was supported by the construction EFOP-3.6.3-VEKOP-16-2017-00002. The project was supported by the European Union, co-financed by the European Social Fund.

\section{References}

[1] Antonini, R.G., Kozachenko, Y. and Volodin, A. Convergence of series of dependent $\varphi$-sub-Gaussian random variables. Journal of Mathematical Analysis and Applications 338 (2008), No. 2, 1188-1203.

[2] Chen, P. Y. and Sung, S. H. A Bernstein type inequality for NOD random variables and applications. Journal of Mathematical Inequalities 11 no. 2 (2017), 455-467.

[3] Bai, Z. D. Methodologies in spectral analysis of large dimensional random matrices. A review. Statistica Sinica, 9 (1999), 611-677.

[4] Bolla, M. Recognizing linear structure in noisy matrices. Linear Algebra and its Applications, 402 (2005), $228-244$.

[5] Bolla, M., Friedl, K. and Krámli, A. Singular value decomposition of large random matrices (for two-way classification of microarrays). Journal of Multivariate Analysis, 101 no. 2 (2010), 434-446. 
[6] Fazekas, I. and Pecsora, S. General Bahr-Esseen inequalities and their applications. Journal of Inequalities and Applications, 2017:191 (2017).

[7] Füredi, Z. and Komlós, J. The eigenvalues of random symmetric matrices. Combinatorica, 1 (1981), 233-241.

[8] The Julia Programming Language, https://julialang.org/

[9] Kahan, W. Spectra of nearly Hermitian matrices. Proceedings of the American Mathematical Society 48 no.1 (1975), 11-17.

[10] Marchenko, V. A. and Pastur, L. A. Distribution of eigenvalues in certain sets of random matrices. (Russian) Matematicheskii Sbornik 72(114) (1967), 507-536.

[11] O'Rourke, S. and Renfrew, D. Low rank perturbations of large elliptic random matrices. Electronic Journal of Probability 19 (2014), paper no. 43, 65 pp.

[12] Tao, T. Outliers in the spectrum of iid matrices with bounded rank perturbations. Probability Theory and Related Fields, 155, 1-2 (2013), 231-263.

[13] Vu, Van H. Spectral norm of random matrices. Combinatorica, 27 (2007), 721-736. 